\title{
Review: primary care counselling improves short term but not long term psychological symptoms in patients with psychological and psychosocial problems
}

\author{
Bower P, Rowland N, Mellor Clark J, et al. Effectiveness and cost effectiveness of counselling in primary care. Cochrane \\ Database Syst Rev 2002;(1):CD001025 (latest version Sep 26 2001).

\section{QUESTION: In patients with psychological and psychosocial problems, is counselling in primary care effective and cost effective?}

\section{Data sources}

Studies were identified by searching databases including Medline; EMBASE/Excerpta Medica; PsycLIT; CINAHL; the Cochrane Library; and the Cochrane Collaboration Depression, Anxiety, and Neurosis Register of Randomized Controlled Trials (RCTs) and Controlled Clinical Trials (for trials completed by June 2001). Search terms included primary health care, counselling, psychotherapy, general practice, and clinical psychology. Bibliographies of relevant studies were scanned, and experts were contacted.

\section{Study selection}

Published and unpublished studies in all languages were selected if they were RCTs; examined patients with psychological or psychosocial problems who were suitable for counselling; used a clear definition of counselling compatible with that of the British Association of Counselling; used counselling offered by qualified, experienced practitioners; included outcomes of clinical effectiveness, patient satisfaction, and health service utilisation; and compared counselling with a control condition. Exclusion criteria were specialist counselling or structured therapies (eg, behavioural therapy and problem solving therapy).

\section{Data extraction}

Data were extracted on methodological quality, participants, type and duration of interventions, psychological and social outcomes, and cost effectiveness. Only the psychosocial and functional outcomes were metaanalysed in this review.

\section{Main results}

7 trials of face to face counselling of individual patients offered by a range of trained counsellors met the selection criteria. Follow up ranged from 6 weeks to 12 months. In 6 trials reporting short term outcomes at 1-6 months $(\mathrm{n}=772)$, patients receiving counselling had better psychological symptom levels than those receiving usual care (pooled standardised mean difference -0.28 , $95 \%$ CI -0.43 to -0.13 at $>6$ months $[\mathrm{n}=475])$. In 4 trials reporting long term outcomes, patients receiving counselling did not differ in psychological symptom levels from those receiving usual care (pooled standardised mean difference $-0.09,95 \%$ CI -0.27 to 0.10$)$. 4 trials reported generally high patient satisfaction levels with counselling. 4 trials reported no clear difference between counselling and usual care for long term costs; however, no power analyses for the economic outcomes were reported, and results were likely underpowered.

\section{Conclusions}

In patients with psychological and psychosocial problems, counselling in primary care improves short term but not long term psychological symptom levels. Patient satisfaction with counselling seems to be high. Data are lacking on the cost effectiveness of counselling.
Sources of funding: Universities of York, Leeds'and Manchester, UK.

For correspondence: Dr P Bower, National Primary Care Research and Development Centre, University of Manchester, Manchester, UK.

peter.bower@man.ac.uk

A modified version of this abstract appears in Evidence-Based

Medicine.

\section{COMMENTARY}

This comprehensive meta-analysis by Bower $e t$ al included the best evidence to answer this important question. This rigour, however, resulted in the inclusion of only 7 studies. The use of experienced counsellors and measurement of psychological and psychosocial outcomes was consistent across studies. A separate sensitivity analysis explored the effect of inadequate allocation concealment in 2 of the 7 studies, but the result did not change when the poorly concealed studies were excluded. Despite the heterogeneity across studies in type and severity of patients, counselling frameworks, and duration of follow up, similar results were found.

Cost effectiveness data were not pooled because of differences in assessing costs across studies. Studies of effectiveness without a rigorous economic analysis can lead to difficulties in interpreting the benefits in the context of the costs incurred. ${ }^{1}$

This review and our own evidence from randomised trials ${ }^{23}$ supports the conclusion of improved short term ( 6 mo tol y) effectiveness and efficiencies achieved with mental health in primary care. Counselling alone without appropriate pharmacotherapy for some will not sustain changes over the long term. Providing counselling and medical care in the same primary care setting facilitates ease of client access to combination therapies.

Gina Browne, RN, PhD
Founder and Director
System-Linked Research Unit on Health and Social Service Utilization
Director, CLEAR Unit
Professor, Nursing and Clinical Epidemiology and Biostatistics
McMaster University
Hamilton, Ontario, Canada

1 Browne G, Roberts J, Gafni A, et al. More effective and less expensive: lessons from five studies examining community approaches to care. Health Policy 1995;34:95-112.

2 Browne G, Roberts J, Gafni A, et al. Economic evaluations of community-based care: lessons from twelve studies in Ontario. J Eval Clin Pract 1999;5:367-85.

3 Browne G, Roberts J, Byrne C, et al. The costs and effects of addressing the needs of vulnerable populations: results of 10 years of research. Can J Nurs Res 2001;33:65-76. 